\title{
Primary cicatricial alopecia: diagnosis and treatment
}

\author{
Rebecca Filbrandt BS, Nicholas Rufaut PhD, Leslie Jones PhD, Rodney Sinclair MD
}

See also practice article by Aslam and Harries on page 1591 and at www.cmaj.ca/lookup/doi/10.1503/cmaj.130305

$\mathrm{H}$ air loss is common, the most prevalent disorders being androgenetic alopecia (male pattern baldness) and alopecia areata. Alopecia areata and androgenetic alopecia are characterized by rapidly cycling miniaturized hair follicles that fail to produce quality terminal hairs. The hair follicle bulbs are not destroyed, so the follicles are able to regenerate and hair regrowth is possible with effective treatment. Androgenetic alopecia affects more than half of men over the age of 50 years. ${ }^{1}$ It is genetic, tends to affect men from their late teen years onward and presents as central or bitemporal recession. About $13 \%$ of premenopausal women and $75 \%$ of women over the age of 65 years experience some androgenetic alopecia, with a typical presentation of thinning over the crown. ${ }^{1}$ Alopecia areata is more commonly associated with acquired thyroid disease, diabetes and collagen diseases, though it is often associated with stress. It affects $0.1 \%-0.2 \%$ of people, with a lifetime risk of $1.7 \% .^{2}$ Smooth round or oval patches of normal skin devoid of hair on the scalp are characteristic of alopecia areata.

In contrast, primary cicatricial alopecia, often referred to as "scarring alopecia," encompasses a group of hair loss disorders in which the hair follicle is irreversibly destroyed and replaced by fibrous tissue. ${ }^{3}$ Hair regeneration is prevented because of destruction of epithelial stem cells in the bulge of the outer root sheath at the level where the arrector pili muscle inserts (Figure 1).,5 Secondary cicatricial alopecia is also irreversible, though destruction of the hair follicle is incidental to a non-follicle-directed cause, such as thermal burns, metastatic cancer, trauma or radiation. ${ }^{4}$ This article will focus on primary cicatricial alopecia.

Primary cicatricial alopecia is not rare, representing about $7 \%$ of patients seen in specialist hair loss clinics. ${ }^{6}$ Clinically, there are 1 or more patches of permanent alopecia on the scalp. These may remain discrete or coalesce to produce a near total alopecia. Within the patches, the skin is bald, smooth and shiny, and pores are absent because of a complete loss of follicular openings. Permanent hair loss can be disfiguring and damage an affected individual's self-esteem. ${ }^{7}$
Primary cicatricial alopecia is challenging, both clinically and therapeutically, and has no known cause. The different forms of primary cicatricial alopecia all share a final pathway of areas of complete hair loss that make it impossible to distinguish many of them from each other. Early disease with active inflammation provides the best opportunity to make a definitive diagnosis; however, changing and overlapping clinical and histological features as the disease evolves also produce diagnostic difficulties. The histological features show greater overlap than the clinical features, and histological diagnosis is less reliable than clinical diagnosis in primary cicatricial alopecia. ${ }^{8}$

In humans, hair follicle neogenesis occurs almost exclusively in utero. In adults, no new hairs form except to a very limited extent following skin wounding. No currently available treatment will stimulate hair follicle neogenesis. As a result, any follicle destroyed by primary cicatricial alopecia will never regrow hair. The goal of treatment is to stop further hair loss and to camouflage the residual bald areas with cosmetics. If there is sufficient undamaged donor hair elsewhere on the scalp, transplantation can be used to cover bald patches, provided the disease is inactive.

Because early diagnosis is essential to the prevention of further hair loss, the purpose of this paper is to review the initial assessment and management of this group of diseases. The evidence used in this review is described in Box 1. Current treatment options to stop hair loss in primary cicatricial alopecia are derived from small
Competing interests: None declared.

This article has been peer reviewed.

Correspondence to: Rebecca Filbrandt, rfilbrandt@gmail.com

CMAJ 2013. DOI:10.1503 /cmaj.111570

\section{KEY POINTS}

- Inflammation that destroys the bulge of the outer root sheath destroys the hair follicle and leads to cicatricial alopecia.

- Primary cicatricial alopecia is an inflammatory disorder of unknown cause that leads to irreversible hair loss.

- The natural history is for the alopecia to extend slowly over the scalp and eventually burn out. The rate of extension and the final severity are extremely variable and difficult to predict.

- A number of treatments are used empirically; however, they are not supported by data from clinical trials. 
case series and consensus guidelines. There is a conspicuous absence of double-blind, placebocontrolled studies. ${ }^{9}$ This is due in part to difficulty measuring the extent of disease and the impact of minimally effective treatments.

\section{How is primary cicatricial alopecia classified?}

A diagnosis provides patients with a better understanding of the prognosis and natural history of their condition. Treatments have evolved empirically based on the nature of the inflammation. The North American Hair Research Society produced a working classification of primary cicatricial alopecia in 2001 that is based on the nature of the predominant inflammatory infiltrate detected on scalp biopsy..$^{10}$ Cases are classified as lymphocytic, neutrophilic or mixed (Table 1)., ${ }^{5,6,11-25}$ In end-stage disease, in which the infiltrate is no longer detectible, the condition is said to be "burned out," and no further classification is possible.

Neutrophilic cicatricial alopecia includes folliculitis decalvans and dissecting cellulitis of the scalp. Folliculitis decalvans is typically seen in adults, though it can affect adolescent males. Dissecting cellulitis of the scalp is rare and occurs predominantly in black adolescent and adult males. ${ }^{3}$ Familial cases of dissecting cellulitis are exceptional, as is childhood onset.

Lichen planopilaris (lichen planus follicularis), chronic cutaneous lupus erythematosus, frontal fibrosing alopecia, acne keloidalis nuchae, central centrifugal cicatricial alopecia and Brocq pseudopelade are lymphocytic forms of alopecia. Lichen planopilaris is the most common cause of cicatricial alopecia and accounts for at least $10 \%$ of cases. ${ }^{6}$ Lichen planopilaris is a clinical variant of lichen planus; both conditions can occur simultaneously. Frontal fibrosing alopecia is a

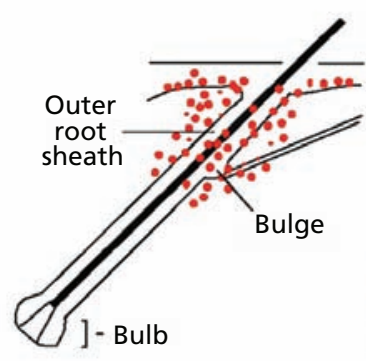

Permanent alopecia

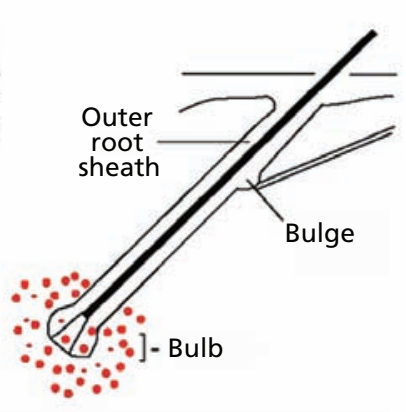

Reversible alopecia
Figure 1: Illustration of the hair follicle. Reversible alopecia (right) is characterized by damage to the hair bulb from inflammation. The hair bulge remains healthy and the hair follicle is able to regenerate with effective treatment. Cicatricial alopecia (left) is characterized by inflammation that causes irreversible damage to the hair bulge of the outer root sheath. variant of lichen planopilaris. The next most common cause of primary cicatricial alopecia in North America is central centrifugal alopecia, which may also be a variant of lichen planopilaris. Folliculitis decalvans and chronic cutaneous lupus erythematosus are other common causes, whereas Brocq pseudopelade is rare.

\section{Which features on history and examination can help in making the diagnosis?}

Because scalp biopsy is often nondiagnostic, a careful history and examination are needed to diagnose primary cicatricial alopecia. Clues to the primary diagnosis can be obtained from age at onset, patient ethnicity, history of skin disease such as lichen planus, lupus or other autoimmune diseases, and previous hair-care practices, along with symptoms and signs such as itching or burning of the scalp and purulent discharge.

Itching of the scalp is an early symptom of lichen planopilaris, and patients may experience pain, patches of rough, scaly skin and blisters. ${ }^{5,9}$ Patients may note purulent discharge in dissecting cellulitis of the scalp and crusting in folliculitis decalvans.

Most forms of primary cicatricial alopecia develop in early adult life. With the exception of 2 rare familial genodermatoses (keratosis folli cularis spinulosa decalvans and Marie Unna hypotrichosis), onset in childhood or adolescence is rare. ${ }^{5,9}$ Frontal fibrosing alopecia occurs in postmenopausal women.

Central centrifugal cicatricial alopecia, acne keloidalis nuchae and dissecting cellulitis of the scalp are found almost exclusively in people of African descent. ${ }^{11}$ Previous hair-care practices such as the use of hot combs, relaxants and excessive traction have been linked to the pathogenesis of central centrifugal cicatricial alopecia, although many affected women have never used these practices. It has been postulated that

\section{Box 1: Evidence used in this review}

We conducted a search of PubMed for literature published from 1962 to Feb. 28, 2013, using the search terms cicatricial alopecia, lichen planopilaris, discoid lupus, pseudopelade primary, androgenetic alopecia, alopecia areata, pathogenesis, classification, lymphocytic, neutrophilic, diagnosis, histopathology, treatment, update and review. We were not able to identify any double-blind, placebocontrolled studies of treatment for primary cicatricial alopecia. We found a number of small case series. Most articles contained detailed descriptions and expert opinion. 


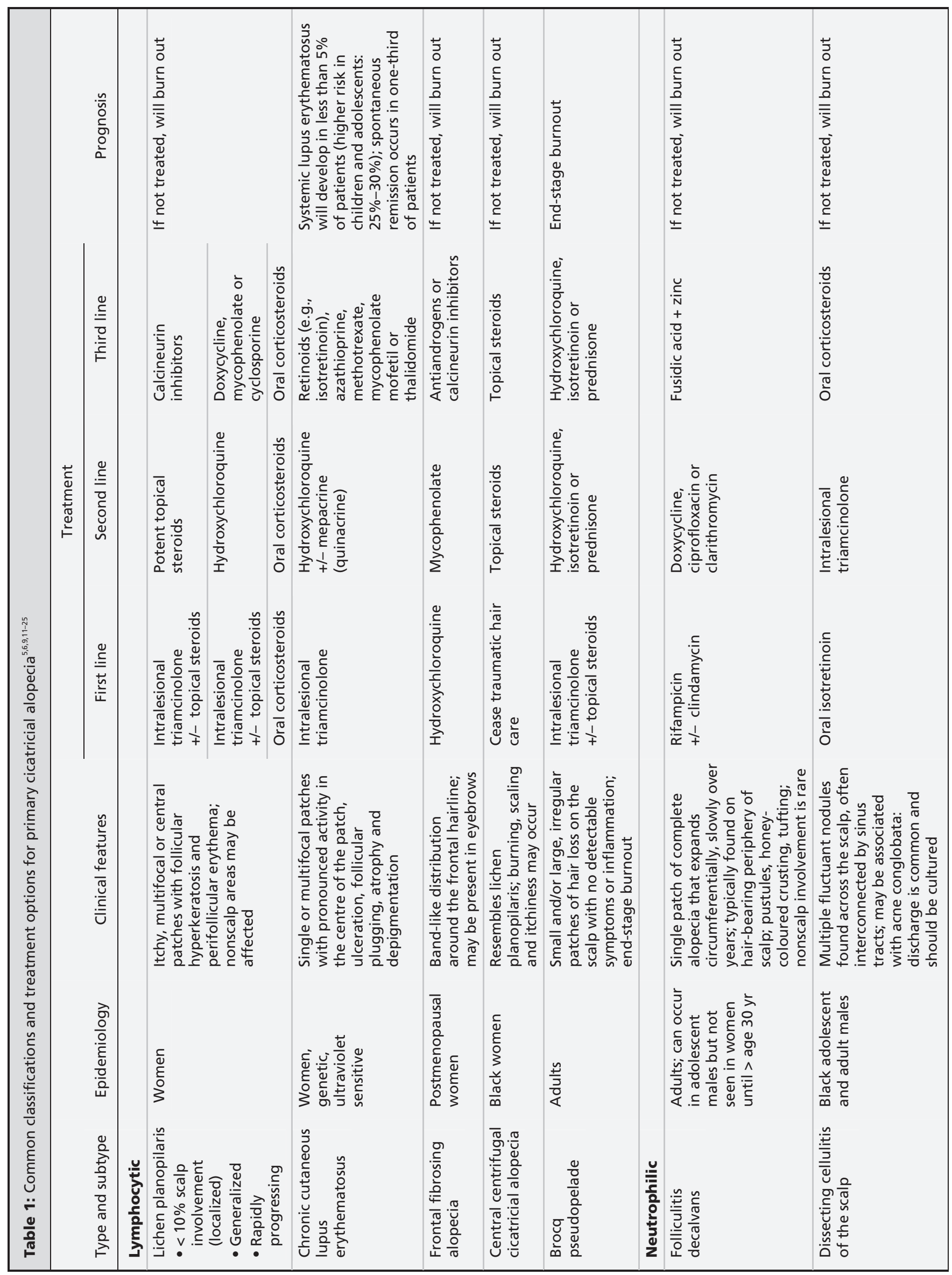


central centrifugal cicatricial alopecia is a cicatrizing form of androgenetic alopecia in women of African descent. ${ }^{12}$

On examination, the first thing to establish is that the hair loss is due to cicatricial alopecia. Magnification may be required to show the absence of pores within the area of hair loss. ${ }^{26}$

\section{Location of lesions}

Central centrifugal cicatricial alopecia and folliculitis decalvans are most common on the vertex and midfrontal scalp. In particular, central centrifugal alopecia may mimic female pattern baldness (see image in Appendix 1, available at www .cmaj.ca/lookup/suppl/doi:10.1503/cmaj.111570 /-/DC1). Acne keloidalis nuchae affects the occipital scalp, predominantly the nape of the neck, whereas frontal fibrosing alopecia (see images in Appendix 2, available at www.cmaj.ca /lookup/suppl/doi:10.1503/cmaj.111570/-/DC1) characteristically affects the frontal hairline with a distinctive band-like recession pattern.,9

Eyebrow alopecia is characteristic in frontal fibrosing alopecia. Typical lesions of chronic cutaneous lupus erythematosus may be seen on the scalp, in the concha of the ear and elsewhere on the face or body in up to $10 \%$ of patients (see
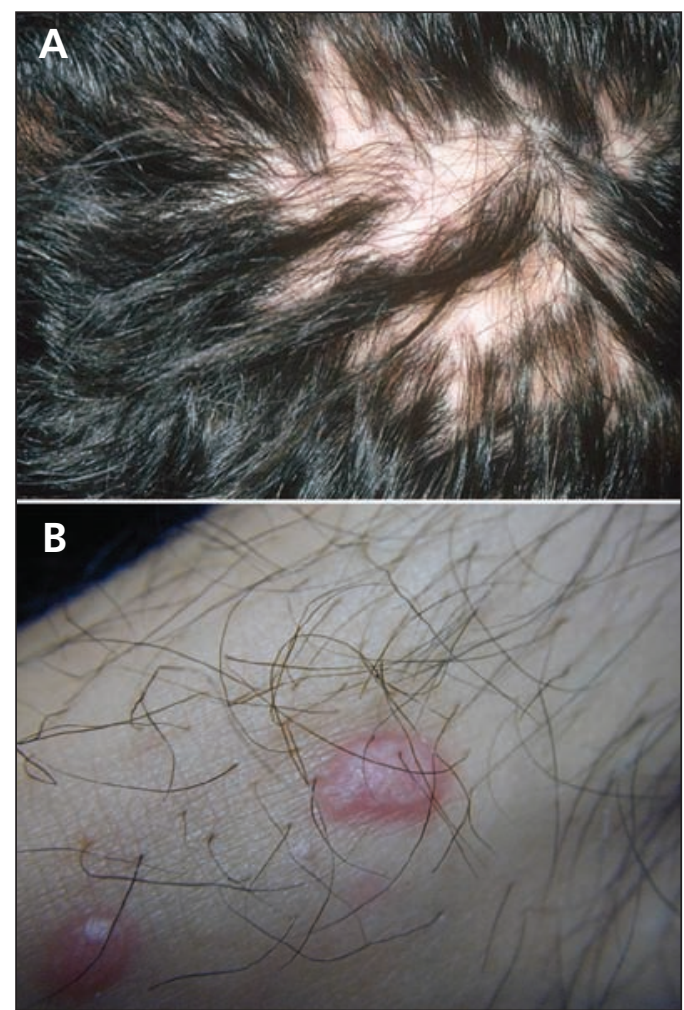

Figure 2: (A) Photograph of a patient's scalp showing lichen planopilaris. Lichen planopilaris is characterized by multifocal or central patches with follicular hyperkeratosis and perifollicular erythema on the scalp. (B) Photograph of a patient's neck showing skin changes with hair growing out of the affected area. images in Appendix 3A and 3B, available at www.cmaj.ca/lookup/suppl/doi:10.1503/cmaj $.111570 /-/ D C 1) .{ }^{14}$

\section{Characteristics of lesions}

Lesions in chronic cutaneous lupus erythematosus show maximal activity in the centre, with perifollicular inflammation, follicular plugging, atrophy and dyspigmentation (see image in Appendix 3C), whereas in lichen planopilaris and folliculitis decalvans, disease activity is most pronounced at the hair-bearing periphery.

Perifollicular erythematous and violaceous papules, spinous follicular hyperkeratosis and multifocal disease support the diagnosis of lichen planopilaris (Figure 2A).,9 The pattern described as "footprints in the snow" with lack of inflammation is suggestive of Brocq pseudopelade (see image in Appendix 4, available at www.cmaj.ca/lookup/suppl/doi:10.1503/cmaj $.111570 /-/ \mathrm{DC} 1)^{5,9}$

In late-stage disease, an important distinguishing feature is that the scar of folliculitis decalvans is thickened, whereas that of lichen planopilaris, chronic cutaneous lupus erythematosus, Brocq pseudopelade and central centrifugal cicatricial alopecia is atrophic.

\section{Associated features}

Skin atrophy is common in lichen planopilaris and central centrifugal cicatricial alopecia. Dyspigmentation is a feature of chronic cutaneous lupus erythematosus and central centrifugal cicatricial alopecia. Nail, oral mucosa or skin changes characteristic of lichen planus are visible at presentation in $17 \%-28 \%$ of patients with lichen planopilaris (Figure 2B)..$^{5,9}$

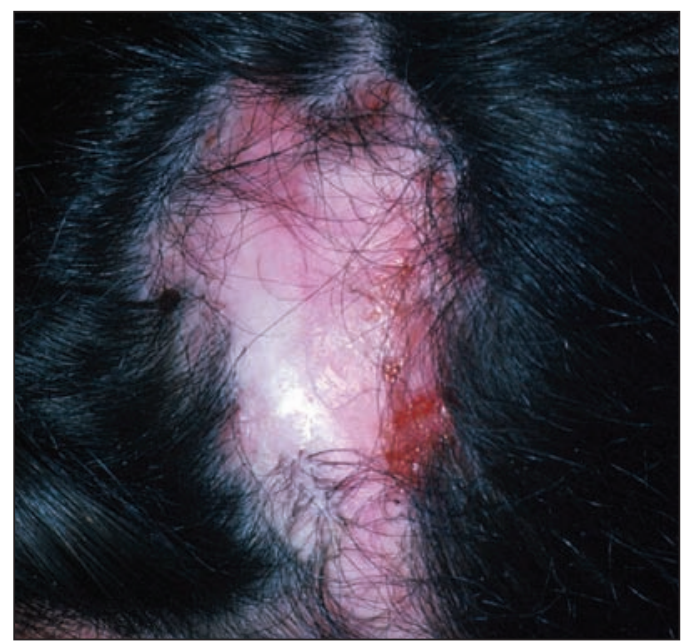

Figure 3: Photograph of a patient's scalp showing folliculitis decalvans. Folliculitis decalvans is characterized by pustules and honey-coloured crusting at the periphery of a patch of hair loss. Patches typically present on the vertex or midfrontal scalp. 
Pustules and honey-coloured crusting at the periphery of a patch of cicatricial alopecia along with tufting of the hair suggest folliculitis decalvans (Figure 3). Purulent discharge, bogginess and sinus tract formation can occur in dissecting cellulitis of the scalp. Dissecting cellulitis of the scalp may also be associated with acne conglobata, pilonidal sinus and hidradenitis suppurativa, as part of the follicular occlusion triad. ${ }^{27}$ Mucinorrhea (release of clear fluid from the pores when squeezed) occurs in alopecia mucinosa.

\section{What investigations may be useful in making the diagnosis?}

A hair pull test, done by gripping about 20 hairs and gently pulling upward and away from the skin allows for the assessment of clinical activity on the scalp. It is normal for about 3 hairs to be extracted with each pull. If more than 10 hairs are removed, the test is considered positive and the hairs should be examined under a microscope to determine hair follicle damage. If crusting, pustules, bogginess or scales are present, hairs should be extracted from the edge of the bald area for microscopy, culture and sensitivity testing (bacterial and fungal), and any pustule should be swabbed and the fluid cultured..$^{13}$ Autoantibodies should be obtained if chronic cutaneous lupus erythematosus is suspected. A hair pull test determines whether sites should be selected for biopsy in the absence of overt inflammation. ${ }^{28}$

Scalp biopsy from the centre of the lesion provides confirmation of permanent hair loss, whereas a biopsy from the edge or an area of active inflammation may shed light on the underlying pathology. ${ }^{29}$ Paired 4-mm punch biopsies for horizontal and vertical sectioning are normally recommended by dermatologists. A third biopsy for direct immunofluorescence may be useful to distinguish between lichen planopilaris and chronic cutaneous lupus erythematosus. ${ }^{5}$ To minimize cross-cutting of the follicles, the biopsy instrument should be positioned parallel to the follicle.

\section{What treatment options are available?}

With the exception of recent-onset chronic cutaneous lupus erythematosus, hair regrowth in primary cicatricial alopecia is not common. Early effective treatment is key to the management of these disorders, with the goal of reducing symptoms and slowing disease progression. Unfortu- nately, treatment response is variable and often incomplete. The variable natural history of these disorders makes it difficult to determine the required duration of treatment. Inability to accurately monitor the response to treatment further compounds the difficulties experienced by clinicians managing these conditions.

There are no placebo-controlled, double-blind randomized trials for the treatment of primary cicatricial alopecia. There are a number of case series and case reports that discuss treatment; however, treatment of these disorders is largely empirical and based on expert opinion. Current treatment recommendations are listed in Table 1. Physicians need to take into account the nature and extent of the disease, rate of progression, potential for adverse effects and patient flexibility (i.e., cost, compliance) in choice of treatment. De novo neogenesis of hair follicles and artificial hair implants are not yet options. ${ }^{30}$

\section{Lymphocytic alopecia}

\section{Lichen planopilaris and frontal fibrosing alopecia}

Lichen planopilaris is generally treated with a combination of topical, intralesional and oral therapies. Topical corticosteroid lotions will control itching and burning. ${ }^{9}$ Potent topical corticosteroid treatment for 12 weeks may halt disease progression but tends to produce substantial atrophy ${ }^{16,17}$ Intralesional injection of corticosteroids (e.g., triamcinolone $10 \mathrm{mg} / \mathrm{mL}$ ) into foci of active inflammation will stop hair loss at the site of injection. Injections may need to be repeated every 6-8 weeks, and care must be taken to avoid re-injecting sites of steroid-induced dermal atrophy..$^{18}$ Calcineurin inhibitors have been tried for localized disease, but the results are often disappointing. ${ }^{31}$

Most patients require systemic therapy. Antimalarials such as hydroxychloroquine are usually used as first-line systemic therapy, with onset of action between 3 and 6 months. The optimal dose is below $7.5 \mathrm{mg} / \mathrm{kg} .{ }^{19,32}$ Possible adverse effects include abdominal pain, anorexia, skin hyperpigmentation, hematologic changes and ophthalmologic damage..$^{33}$ Regular monitoring for the development of retinopathy should be started.

Oral corticosteroids (e.g., $25-40 \mathrm{mg} / \mathrm{d}$ of prednisone for 2-4 mo) are reserved for rapidly progressing and severely symptomatic disease. In patients unable to tolerate oral corticosteroids, cyclosporine (3-5 mg/kg for 3-5 d) may be effective. ${ }^{33}$

Oral retinoids are effective in some people but are poorly tolerated because of their tendency to induce hair shedding. Other options reported in 
small case series include doxycycline (100 mg twice daily) and mycophenolate (500 mg twice daily for $1 \mathrm{mo}$ and then $1 \mathrm{~g}$ twice daily for $5 \mathrm{mo}$ ). ${ }^{3.33}$

The management of the frontal variant is similar to ordinary lichen planopilaris. Chiang and colleagues $^{19}$ and Cho and colleagues ${ }^{20}$ reported benefit with the use of hydroxychloroquine and mycophenolate. The use of antiandrogens and calcineurin inhibitors has also been advocated. ${ }^{21}$

Recently, the asebia mouse, with an autosomal recessive trait resulting in hypoplastic sebaceous glands, has been shown to develop hair loss that shares many features with lichen planopilaris. ${ }^{3}$ The mouse develops a progressive alopecia with sparse or matted hair, pruritus and scaly skin that shares many clinical and histological features with primary cicatricial alopecia. This has led to the hypothesis that sebaceous gland dysfunction contributes to the development of primary cicatricial alopecia, possibly through impaired breakdown of the inner root sheath. ${ }^{34}$ The hair loss in the asebia mouse responds partially to peroxisome proliferatoractivated receptor $\gamma$ agonist medications, such as thiazolidinedione or glitazone medications, which are widely used for the treatment of type 2 diabetes mellitus. Based on these findings in mice, clinicians have successfully treated refractory lichen planopilaris using pioglitazone hydrochloride ${ }^{35}$ Although this is a potentially important development, confirmatory case reports and clinical trials are needed.

\section{Chronic cutaneous lupus erythematosus}

Chronic cutaneous lupus erythematosus is known to progress with exposure to ultraviolet light, and thus protection of the scalp with a hat, and face and body with broad-spectrum sunscreen should be used on a daily basis. ${ }^{15}$ Topical steroids may be effective in many cases, whereas intralesional triamcinolone is effective in most cases. ${ }^{22}$ For people with widespread or refractory disease, however, hydroxychloroquine should be considered. Patients usually require $400 \mathrm{mg}$ daily during the summer months, but the dose can be reduced during the cooler seasons. The efficacy of hydroxychloroquine appears to be reduced with smoking, and patients who smoke should be encouraged to stop. ${ }^{36}$

In patients with recent-onset, rapidly progressive, severe or refractory disease, oral prednisolone (e.g., $0.5-1 \mathrm{mg} / \mathrm{kg}$ tapered over 8 wk) may be considered. ${ }^{18}$ Patients with recent-onset disease may experience a surprising degree of regrowth with early aggressive treatment.

For patients with chronic disease, a second antimalarial, mepacrine (quinacrine), can be added if the response to hydroxychloroquine is unsatisfactory after 6 months of therapy. In patients with resistance to antimalarials, oral retinoids could be used. The response to oral retinoids is often rapid, but the risk of teratogenicity should be considered. Telogen effluvium, drug-induced hair loss that causes the hair follicle to enter the resting phase (telogen) and fall out prematurely, may occur within 6 months of treatment with oral retinoids. The risk of telogen effluvium has been shown to be substantially lower with the use of isotretinoin rather than acitretin. ${ }^{9}$ Azathioprine, methotrexate, mycophenolate mofetil and thalidomide have also been used in treating chronic cutaneous lupus erythematosus. ${ }^{9}$

\section{Other types of lymphocytic alopecia}

No treatment has been shown to influence Brocq pseudopelade or central centrifugal cicatricial alopecia. Alopecia mucinosa may respond to topical or intralesional corticosteroids, phototherapy or superficial radiotherapy. ${ }^{5}$

\section{Neutrophilic alopecia}

\section{Folliculitis decalvans}

Folliculitis decalvans is most often treated with antibiotics and antiseptics. All patients with folliculitis decalvans should use an antiseptic shampoo. Topical benzoyl peroxide or topical clindamycin will control mild cases. For severe or refractory cases, oral antibiotics will be required. ${ }^{6}$ The ability of an antibiotic to achieve adequate bactericidal concentration within hair follicles is an important consideration. The most common bacteria identified is Staphylococcus aureus. If pustules are seen, they should be swabbed and the antibiotic chosen based on the culture and antibiotic sensitivity test. ${ }^{3}$

Commonly, there are no active pustules or crusts to swab, and antibiotics are chosen empirically. Minocycline is commonly used and will control many cases. The combination of rifampicin (300 mg twice daily), clindamycin (300 mg twice daily) and fusidic acid together with topical corticosteroid lotion for 3 months has been used with success. ${ }^{23,24}$ Rifampicin, in particular, achieves high concentration within hair follicles; however, it should not be used as monotherapy, because this promotes emergence of resistant organisms. Prolonged use of oral clindamycin can lead to diarrhea, and so topical use may be preferred. Minomycin, dicloxacillin, ciprofloxacin and clarithromycin have also been used to treat folliculitis decalvans. ${ }^{3,24} \mathrm{~A}$ few patients have been known to respond well to oral isotretinoin; however, many patients experience flares with this treatment. ${ }^{23}$ It should be reserved for refractory cases. 


\section{Dissecting cellulitis}

In contrast to folliculitis decalvans, dissecting cellulitis responds well to oral isotretinoin (0.5$1 \mathrm{mg} / \mathrm{kg}$ daily). Treatment for 6-11 months allows lesions to shrink and has resulted in prolonged periods of remission. ${ }^{25}$

Hair regrowth in areas not yet irreversibly damaged is possible in some cases and may be assisted by oral or intralesional corticosteroids. ${ }^{6.13}$

\section{Surgery}

In general, surgery is useful only for patients with lesions that are stable in size and for whom the inflammation driving the hair loss has been burned out for at least 1 year. Scalp reduction surgery, which can be complemented by hair transplantation, is most effective. ${ }^{37}$

\section{Gaps in knowledge}

Future investigations must move from morphological descriptions to molecular ones, identifying antigens or cell-bound targets of the infundibulum and isthmus and identifying triggers for the folliculocentric attack. A number of mouse models for cicatricial alopecia have now been identified and will help identify new disease mechanisms and therapeutic targets. ${ }^{4}$ Improvements in the safety of artificial hair fibres for transplantation are required before they can be recommended. ${ }^{38,39}$ Methods to expand donor tissue for autologous hair transplantation through hair cloning are under investigation.

\section{References}

1. Harrison S, Bergfeld W. Diffuse hair loss: its triggers and management. Cleve Clin J Med 2009;76:361-7.

2. Petukhova L, Duvic M, Hordinsky M, et al. Genome-wide association study in alopecia areata implicates both innate and adaptive immunity. Nature 2010;466:113-7.

3. Harries MJ, Sinclair RD, Macdonald-Hull S, et al. Management of primary cicatricial alopecias: options for treatment. $\mathrm{BrJ}$ Dermatol 2008;159:1-22.

4. Harries MJ, Paus R. The pathogenesis of primary cicatricial alopecias. Am J Pathol 2010;177:2152-62.

5. Harries M, Trueb RM, Tosti A, et al. How not to get scar(r)ed: pointers to the correct diagnosis in patients with suspected primary cicatricial alopecia. Br J Dermatol 2009:160:482-501.

6. Whiting DA. Cicatricial alopecia: clinico-pathological findings and treatment. Clin Dermatol 2001;19:211-25.

7. Cash TF. The psychosocial consequences of androgenetic alopecia: a review of the research literature. Br J Dermatol 1999; 141:398-405.

8. Mirmirani P, Willey A, Headington JT, et al. Primary cicatricial alopecia: Histopathologic findings do not distinguish clinical variants. J Am Acad Dermatol 2005;52:637-43.

9. Ross EK, Tan E, Shapiro J. Update on primary cicatricial alopecias. J Am Acad Dermatol 2005;53:1-37.

10. Olsen EA, Bergfield WF, Costarelis G, et al. Summary of North American Hair Research Society (NAHRS)-sponsored workshop of cicatricial alopecia. Duke University Medical Center, February 10-11, 2001. Am Acad Dermatol 2003:48:103-10.

11. Sperling LC, Sau P. The follicular degeneration syndrome in black patients. "Hot comb alopecia" revisited and revised. Arch Dermatol 1992;128:68-74.

12. Olsen EA, Callender V, McMichael A, et al. Central hair loss in
African American women: incidence and potential risk factors. J Am Acad Dermatol 2011;64:245-52.

13. Sinclair RD. Acquired cicatricial alopecias. In: Burns DA, Breathnach SM, Cox NH, et al., editors. Rook's textbook of dermatology. 7th ed. Oxford (UK): Blackwell Publishing; 2004. p. 63.46-63.61

14. Pramatarov KD. Chronic cutaneous lupus erythematosus clinical spectrum. Clin Dermatol 2004;22:113-20.

15. Werth V. Current treatment of cutaneous lupus erythematosus. Dermatol Online J 2001;7:2

16. Chieregato $\mathrm{C}$, Zini A, Barbara A, et al. Lichen planopilaris: report of 30 cases and review of the literature. Int $J$ Dermatol 2003; $42: 342-5$.

17. Mehregan DA, Van Hale HM, Muller SA. Lichen planopilaris: clinical and pathologic study of forty-five patients. J Am Acad Dermatol 1992;27:935-42.

18. Tan E, Martinka M, Ball N, et al. Primary cicatricial alopecias: clinicopathology of 112 cases. J Am Acad Dermatol 2004;50:25-32.

19. Chiang C, Sah D, Cho BK, et al. Hydroxychloroquine and lichen planopilaris: efficacy and introduction of Lichen Planopilaris Activity Index scoring system. J Am Acad Dermatol 2010; 62:387-92.

20. Cho BK, Sah D, Chwalek J, et al. Efficacy and safety of mycophenolate mofetil for lichen planopilaris. J Am Acad Dermatol 2010;62:393-7.

21. Katoulis A, Georgala, Bozi E, et al. Frontal fibrosing alopecia: treatment with oral dutasteride and topical pimecrolimus. J Eur Acad Dermatol Venereol 2009;23:580-2.

22. Rowell NR. Treatment of chronic discoid lupus erythematosus with intralesional triamcinolone. Br J Dermatol 1962;74:354-7.

23. Powell J, Dawber RP. Successful treatment regime for folliculitis decalvans despite uncertainty of all aetiological factors. $\mathrm{Br} \mathrm{J}$ Dermatol 2001;144:428-9.

24. Powell J, Dawber RP, Gatter K. Folliculitis decalvans including tufted folliculitis: clinical, histological and therapeutic findings. Br J Dermatol 1999;140:328-33.

25. Scerri L, Williams HC, Allen BR. Dissecting cellulitis of the scalp: response to isotretinoin. Br J Dermatol 1996;134:1105-8.

26. Sperling LC. Evaluation of hair loss. Curr Probl Dermatol 1996; 8:101-36.

27. Scheinfeld NS. A case of dissecting cellulitis and a review of the literature. Dermatol Online J 2003;8:1.

28. Chong AH, Wade M, Sinclair RD. The hair pull test and the hair pluck test for the analysis of hair abnormalities. Mod Med 1999; 42:105-8.

29. Shapiro J. Cicatricial alopecias. Dermatol Ther 2008;21:211.

30. Paus R. Therapeutic strategies for treating hair loss. Drug Discov Today Ther Strateg 2006;3:101-10.

31. Walker SL, Kirby B, Chalmers RJ. The effect of tacrolimus on severe recalcitrant chronic discoid lupus erythematosus. $\mathrm{Br} \mathrm{J}$ Dermatol 2002; 147:405-6.

32. Van Beek MJ, Piette MM. Antimalarials. Dermatol Clin 2001; 19:147-60.

33. Price VH. The medical treatment of cicatricial alopecia. Semin Cutan Med Surg 2006;25:56-9.

34. Al-Zaid T, Vanderweil S, Zembowicz A, et al. Sebaceous gland loss and inflammation in scarring alopecia: a potential role in pathogenesis. J Am Acad Dermatol 2011;65:597-603.

35. Mirmirani P, Karnik P. Lichen planopilaris treated with a peroxisome proliferator-activated receptor gamma agonist. Arch Dermatol 2009; 145:1363-6.

36. Jewell ML, McCauliffe DP. Patients with cutaneous lupus erythematosus who smoke are less responsive to antimalarial treatment. J Am Acad Dermatol 2000;42:983-7.

37. Unger WP. Hair transplantation: current concepts and techniques. J Investig Dermatol Symp Proc 2005;10:225-9.

38. Lange-Ionescu S, Frosch PJ. Complications of synthetic hair implantation. Hautarzt 1995;46:10-4.

39. Agrawal M. Modern artificial hair implantation: a pilot study of 10 patients. J Cosmet Dermatol 2008;7:315-23.

Affiliations: American University of the Caribbean School of Medicine (Filbrandt), Cupecoy, St. Maarten; Department of Dermatology (Rufaut, Jones, Sinclair), Epworth Hospital Melbourne; Departments of Medicine (Rufaut, Jones, Sinclair), University of Melbourne, St. Vincent's Hospital, Melbourne, Australia

Contributors: All of the authors contributed to the conception and design, drafting and revision of the article and approved the final version submitted for publication. 